\title{
Staphylococcus epidermidis and retention of neonatal percutaneous central venous catheters
}

\author{
G Gladman, S Sinha, D G Sims, M L Chiswick
}

\begin{abstract}
The percutaneous insertion of central venous catheters has become an established practice on many neonatal units. We describe four low birthweight babies, whose catheters became tethered in the vein, and discuss the management of this unusual complication.
\end{abstract}

Central venous catheterisation to facilitate total parenteral nutrition in sick very low birthweight babies avoids the need for repeated insertion of cannulas into peripheral veins. It also reduces the incidence of scarring associated with prolonged infusion of hypertonic solutions through small blood vessels.

Percutaneous central venous catheterisation, first described by Shaw in $1973,{ }^{1}$ is commonly used because it avoids the need for surgical insertion under general anaesthesia. We report four babies in whom a percutaneously inserted central venous catheter became retained in the vein, requiring surgical removal in two. We indicate the possible role of septic thrombus formation in tethering central venous catheters and suggest guidelines for the management of this complication.

\section{Case reports}

In each of the four cases described, a standard percutaneous central venous catheter was used (radio-opaque silastic central venous catheter, $0.3 \mathrm{~mm}$ internal diameter, $0.6 \mathrm{~mm}$ external diameter, Vygon Ltd) and all were inserted using strict aseptic technique. Catheter related sepsis was only conclusively diagnosed when the same organism was recovered from both the catheter and blood in an unwell baby. Microbiological assessment employed both semiquantitative culture techniques (rolling the catheter tip across culture medium) and immersion in liquid broth, in an attempt to distinguish 'contamination' from 'true infection'.

North Western Regional

Perinatal Centre,

St Marys Hospital,

Manchester

G Gladman

S Sinha

D G Sims

M L Chiswick

Correspondence to:

Dr G Gladman, Booth Hall Children's Hospital, Charlestown Road, Blackley, Manchester M9 2AA

Accepted 17 October 1989
CASE 1

A boy, weighing $1705 \mathrm{~g}$, born at 29 weeks' gestation suffered severe respiratory distress, bilateral pneumothoraces, and required a prolonged period of mechanical ventilation. To facilitate the administration of total parenteral nutrition, a fine silastic feeding catheter was introduced on day 17 through a vein on the left side of the scalp. The catheter tip was confirmed to be in the right atrium on radiography and intravenous feeding was uneventful until day 33 when Staphylococcus epidermidis septicaemia occurred and removal of the catheter was considered necessary. On moderate traction, the catheter appeared tethered and firmer efforts led to the catheter snapping. By measuring the length of catheter removed, it was apparent that a $4 \mathrm{~cm}$ portion of catheter had been retained. The remnant could be palpated through the skin overlying the left side of the scalp, just distal to the original site of entry of the cannula. Through a small incision in the left temple, the remnant of the catheter was successfully removed and radiography confirmed complete removal of the cannula. On microbiological culture, both blood cultures from the infant and the retained segment showed a significant growth of $S$ epidermidis.

CASE 2

The second of twin girls born at 29 weeks' gestation, weighing $1060 \mathrm{~g}$, required prolonged ventilation for severe respiratory distress. A percutaneous central venous catheter was inserted via a vein on the dorsum of the right hand on day 12. The tip was confirmed to be in the right atrium on radiography and total parenteral nutrition was uneventful until day 25 when the infant became acutely unwell. There was slight erythema around the site of insertion of the catheter and its removal was considered necessary. The catheter, however, could not be withdrawn. Steady, continuous traction was applied to the catheter by pulling it to 'full stretch' and then taping it in this position to a splint attached to the baby's arm (figure). Every few hours, the catheter was 'restrapped' to maintain maximum traction. After 36 hours the catheter became free and complete removal was achieved. Blood cultures from the infant showed a significant growth of $S$ epidermidis, but the catheter tip failed to show any growth in this case.

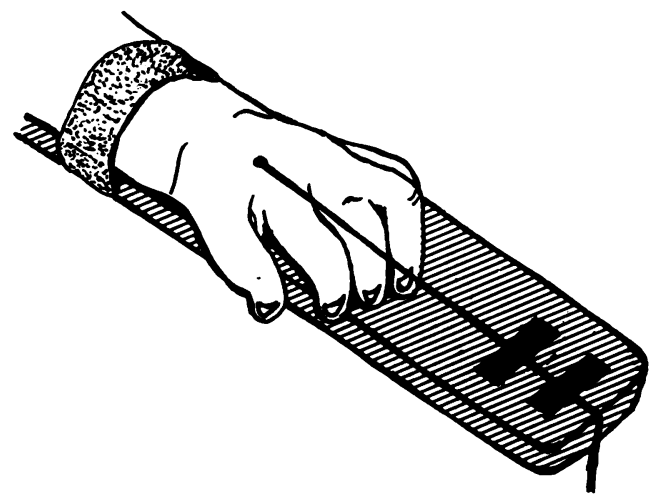

The splint traction arrangement. 
CASE 3

A percutaneous central venous catheter was introduced through a vein in the right antecubital fossa on day 24 of life in a girl born at 26 weeks' gestation weighing $700 \mathrm{~g}$. The tip of the catheter was confirmed to be in the right atrium on radiography. After only five days, the infant became unwell and the limb became increasingly swollen and removal of the catheter was attempted. On traction the catheter offered firm resistance and then snapped leaving a retained portion. Radiography showed $7 \mathrm{~cm}$ of retained catheter, with the tip still in the right atrium and the proximal end fixed at the level of the deltopectoral groove. Surgical exploration at the latter site enabled the remaining catheter to be removed and complete removal was confirmed on radiography. Both the catheter tip and blood cultures from the baby yielded a significant growth of $S$ epidermidis.

\section{CASE 4}

The first of triplets, born at 26 weeks' gestation and weighing $800 \mathrm{~g}$, required prolonged ventilatory support. To facilitate total parenteral nutrition a silastic central venous catheter was introduced on day 30 of life. Having confirmed the catheter to be in a satisfactory position on radiography, intravenous feeding was uneventful until day 44 of life, when the infant became acutely unwell and removal of the catheter was considered necessary. The catheter appeared firmly tethered in the vein, however, and could not be withdrawn. Traction was applied using the splint arrangement described earlier, and after 18 hours the catheter became free and complete removal was achieved. Blood cultures from the infant and the catheter tip both yielded a heavy growth of $S$ epidermidis.

\section{Discussion}

Although many neonatal units have probably observed retention and snapping of percutaneously inserted central venous catheters, we could find no reports of this particular complication in the literature and few guidelines on the management of this problem.

Recent papers have described the method of percutaneous silastic catheter insertion and reported other catheter associated complications-mainly occlusion of the line, infection, and limb swelling. ${ }^{2}{ }^{3}$ In these reports the mean duration the catheters were in situ was 17 days $^{2}$ and 34 days. $^{3}$ While sepsis was the most important complication, both studies reported a comparatively low incidence of catheter related bacteraemia (13\% and $8 \%$ ) compared with that from surgically placed catheters ( $18 \%$ infected). ${ }^{4}$ During the 18 month period in which these cases occurred, approximately 50 babies under- went percutaneous central venous catheterisation in our hospital. Although we observed proved catheter related sepsis (same organism cultured from both catheter and blood) in at least two other infants during this time interval, we did not observe tethering of the catheter in any infant who did not show signs, both clinically and microbiologically, of $S$ epidermidis septicaemia. Retention of the catheter in our infants was not associated with a particular site of insertion. All the catheters were well within the manufacturers' recommended expiry date, and none were unduly difficult to insert. In none of our infants was the duration of cannulation as long as the mean duration reported in the above studies. Septic thrombus formation leading to tethering of the catheter in the vein seems a likely aetiological factor.

All of the infants described made a full recovery and fortunately none of the catheter remnants broke free into the circulation, where the potential complications might include massive intracardiac thrombosis, pulmonary embolism, erosion of the right atrial or ventricular wall with subsequent haemopericardium, and mycotic or bacterial endocarditis.

In the light of our experience with these babies, we would suggest that if a central venous catheter becomes tethered and cannot easily be removed, firm continuous traction using the splint arrangement described in the text may help to 'release' the catheter. If a catheter snaps, an immediate radiograph of the appropriate site will determine the position of the remnant (assuming the catheter is radioopaque) and once the best site for surgical exploration has been decided, the retained segment should be removed as soon as possible. It is important to ascertain that complete removal of the catheter has been achieved after surgery-either by measuring the catheter pieces or repeating the radiograph. In larger infants it may be possible to retrieve catheter remnants from the large vessels and from an intracardiac site using cardiac catheterisation techniques $^{5}$ and so avoid surgery. Finally, we would recommend notifying the manufacturing company, as they are always interested to examine any catheters that have 'behaved abnormally'.

1 Shaw JCL. Parenteral nutrition in the management of sick low birthweight infants. Pediatr Clin North Am 1973;20. 333-58.

2 Puntis JWL. Percutaneous insertion of central venous feeding catheters. Arch Dis Child 1986;61:1138-40.

3 Ramanathan R, Durand $M$. Blood cultures in neonates with percutaneous central venous catheters. Arch Dis Child 1987;62:621-3.

4 Mactier R, Alroomi LG, Young DG, Raine PAM. Central venous catherisation in very low birthweight infants. Arch Dis Child 1986;61:449-53.

5 Gross DM, Cox MA, Denson SB, Ferguson L. Unique use of a tip-deflecting guide wire in removing a catheter embolus from an infant. Pediatr Cardiol 1987;8:117-9. 